Article

\title{
Numerical Study of the Effect of Winglets on the Performance of a Straight Blade Darrieus Water Turbine
}

\author{
Santiago Laín ${ }^{1, *}$, Manuel A. Taborda ${ }^{1}$ and Omar D. López ${ }^{2}$ \\ 1 Modelling, Analysis and Simulation of Environmental and Industrial Processes (PAI+) Research Group, \\ Universidad Autónoma de Occidente, Calle 25 No 115-85, Cali, Colombia; matabordac@gmail.com \\ 2 Computational Mechanics Research Group, Universidad de los Andes, Carrera 1Este No 19A-40, Bogotá, \\ Colombia; od.lopez20@uniandes.edu.co \\ * Correspondence: slain@uao.edu.co
}

Received: 11 October 2017; Accepted: 10 December 2017; Published: 28 January 2018

\begin{abstract}
This study deals with the three-dimensional unsteady numerical simulation of the flow around a cross-flow vertical-axis water turbine (CFWT) of the Darrieus type. The influence of turbine design on its hydrodynamic characteristics and performance is investigated by means of a time-accurate Reynolds Averaged Navier Stokes (RANS) commercial solver. The flow unsteadiness is described using a transient rotor-stator model in connection with a sliding interface. A classical Darrieus straight blade turbine, based on the NACA0025 airfoil, has been modified adding winglets (symmetric and asymmetric designs) to the blades' tips with the objective of reducing the strength of the detached trailing vortices. The turbulent features of the flow have been modelled by using different turbulence models ( $k-\varepsilon$ Renormalization Group, standard Shear Stress Transport, transition Shear Stress Transport and Reynolds Stress Model). As a result, the predicted hydrodynamic performance of the turbine including winglets increases, independently of the employed turbulence model, being the improvement higher when a symmetric winglet design is considered. Moreover, visualization of skin friction lines pattern and their connection with vorticity isosurfaces, illustrating the flow detachment in the three blade configurations, has been carried out. Finally, a short discussion about the intermittency behavior along a turbine revolution is presented.
\end{abstract}

Keywords: CFD simulation; transient analysis; water turbine; turbulence model; winglets

\section{Introduction}

The pollution issues created by the consumption of fossil fuels and their limited availability, together with concerns about environmental problems, have triggered the quest for alternative renewable energy sources. An option with high chances of success is the exploitation of the kinetic energy of marine currents. For harvesting this energy two types of turbines are mainly employed: horizontal axis turbine (HAWT), whose rotation axis is parallel to the incoming flow; and vertical axis turbines, known also as cross flow water turbine (CFWT) because their axis of rotation is perpendicular to the stream. The main advantage of the second type is the absence of an orientation mechanism, although its efficiency is lower than that of the horizontal axis machines.

The flow around vertical turbine is considerably more complex than around horizontal turbines as in the former the flow around the blades is intrinsically unsteady, presents rapidly changing angles of attack that generate detached vortices and wakes that interact with the other blades. Therefore, simplified design and evaluation strategies based on potential methods have had limited success, especially for high turbine solidities [1]. As an alternative to describe the flow around CFWTs, Computational Fluid Dynamics (CFD), a well-established tool in academic and industrial sectors [2-6], 
started to be used since the beginning of this century, first as simplified two-dimensional models of straight blade Darrieus turbines and, only recently, using full three-dimensional models. A review of the simplified approach strategies for CFD simulation of CFWTs was performed in [7].

The reason why three-dimensional CFD computations of straight blade Darrieus turbines started only recently was the need of powerful and fast computers. The first attempts dealt essentially with wind turbines, due to their extended use to generate electricity. Some of such studies are reviewed in the following.

Howell et al. [8] conducted an experimental and computational research on the performance of a low aspect ratio (i.e., four) Darrieus wind turbine (VAWT) with three blades. The numerical simulations were carried out with the commercial ANSYS-Fluent software in two and three dimensions employing the Reynolds Averaged Navier Stokes (RANS) methodology based on the $k$ - $\varepsilon$ RNG turbulence model. In this work very coarse grids were used for the 3D computations (around 1.3 million cells) as wall functions were employed. According to the numerical simulations, the estimated power coefficient in the 2D geometry was substantially higher than the obtained in the 3D geometry and the experiments. The authors claimed that such overestimation was due to the absence of detached tip vortices that cannot be captured by the two-dimensional simulations.

Untaroiu et al. [9] studied the self-starting behavior of a Durham type wind turbine with three blades [10], employing the ANSYS-CFX solver and $k-\varepsilon$ turbulence model. As a result, two-dimensional $\left(1.36 \times 10^{5}\right.$ cells $)$ and three-dimensional $\left(7.9 \times 10^{6}\right.$ cells $)$ grids provide similar final angular velocity. However, the acceleration of the turbine is overestimated by an order or magnitude, which was attributed to the poor wall treatment of the turbulence model, although the $y^{+}$values in both grids were below 10. In this work only the three rotating blades were included in the geometrical model.

The effect of inclination angle on power was numerically investigated by Castelli and Benini [11] in the case of a one blade helical Darrieus wind turbine. In this study, the influence of the turbulence model on results was explored, although the $y^{+}$values obtained in the 3D simulation were not reported. Performance of $k-\omega$ SST and $k-\varepsilon$ realizable models was very similar and both provided higher torque coefficients than the Spalart-Allmaras model. However, no comparison with experimental data was provided in this work.

Siddiqui et al. [12] numerically quantified the effects of the geometric modeling on the performance of a vertical axis wind turbine. They evaluated several approximations: 2D computations, $2.5 \mathrm{D}$ approximation (i.e., extrusion of the $2 \mathrm{D}$ grid with symmetry boundary condition at the blades span), 3D geometry considering blades only and 3D simulation with blades, shaft and arms. In all cases fairly coarse grids are used and the $k-\varepsilon$ realizable turbulence model is employed. In their computations, they found that the 2D approximation can over-estimate the power coefficient up to $32 \%$ regarding the full 3D computation, while neglecting the support structure can induce an over-estimation of $11 \%$ in the value of the same coefficient.

Regarding the performance prediction of Darrieus water turbines, Laín and Osorio [13] showed two-dimensional numerical results for the torque coefficient of a CFWT, reported by [14] using CFD that are close to the experimental data. Amet et al. [15] performed a very detailed 2D simulation of two-bladed CFWT and compared with experimental data of several authors for two tip speed ratios (TSR) of 2 and 7. In this paper a very refined structured grid is used around the airfoils with a value of $y^{+}$around 1 . An extensive discussion about the blade-vortex interaction is developed in this paper concluding that the flow in the case of the smaller tip speed ratio was characterized by a deep stall regime with several large vortices detached from the blades while for the higher TSR the flow is characterized by a weak shedding of alternated vortices in the upstream half disk and by an attached flow in the downstream half disk.

Maître et al. [16] performed detailed two dimensional simulations of the three blades model CFWT LEGI available in their laboratory. The influence of the grid refinement close to the blade surface on the mean power extracted is discussed and analyzed. The main conclusions obtained by the authors were: (1) The obtained mean power decreases fast when the grid coarsens in the proximity of the 
profiles surface; (2) A coarse mesh induces an early and severe loss of lift due to the formation of a back flow bubble, which generates unrealistic pressure drag. It was also observed that the predicted power coefficient is notably higher than the experimental values, except for very low tip speed ratios where computations are below the measurements. This observation is in agreement with other authors found in the literature. This work also cites the three-dimensional simulations of [17] on the same water turbine performed for just one tip speed ratio employing a grid with 8 million nodes. In such a case the computed power coefficient was very close to the measured value at best efficiency point. In this last study also it is mentioned that the influence of the detached tip blade trailing vortices on the turbine performance loss is around $22 \%$.

Hall [18] developed a methodology for numerical modeling of helical-bladed crossflow (vertical) water turbines. The sliding mesh model together with a $k-\omega$ SST turbulence model were employed to simulate a four-blades rotating turbine. Excellent qualitative results and good quantitative agreement with the measured values were found; however, known RANS limitations associated with prediction of flow separation and stall phenomenon are responsible for some discrepancies between the computed and measured values, especially for the smallest values of the tip speed ratio.

Pellone et al. [19] report three-dimensional computations also on the LEGI water turbine including the cylindrical hub and the three arms, reproducing the complete experimental model. The computational mesh had more than five million cells. The predicted averaged power coefficient for a tip speed ratio of 2 was very close to the experimentally measured value and much lower than the values predicted by the two-dimensional model and a 3D model considering only the blades. However, the instantaneous power coefficient curves, although similar, differ quantitatively; in particular the power peak at $90^{\circ}$ angle is over-estimated by the computations which is compensated by a power under-prediction at a higher angle. Also the presence of an interference vortex at the blade-arm junction is clearly identified in such paper. Such a vortex significantly affects the torque distribution along the blade span. These facts illustrate the complexity of the flow around cross-flow water turbines. The computational study of [20] presents the influence of blade helicity on the performance of vertical axis tidal turbines. In particular they study two designs of straight blade turbines differing in the blade-strut connection. In the so-called $0^{\circ}$ turbine the blade-arm connection is at the blade tips, working as a sort of tip device, while in the so-called $0^{\circ}$-A turbine the struts connect the blade at $1 / 4$ of the span. As a main result (numerical and experimental) the maximum power coefficient of the $0^{\circ}$ model is close to double than that of the $0^{\circ}$-A model. The main conclusions found by Marsh et al. are that straight-bladed turbines provide better performance than helical turbines, but the latter reduce more effectively the torque oscillation levels and mounting forces.

Regarding the influence of turbulence modelling on performance of vertical axis turbines, both for wind and water applications, several studies can be found in the literature. For example, Ferreira [21] performed 2D and 3D simulations of a vertical axis wind turbine and compared the numerical results with experimental data including PIV. Different turbulence models such as Spalart-Allmaras (SA), $k-\varepsilon$, LES and Detached Eddy Simulation (DES) were tested. Numerical results showed that the URANS models that were tested (SA and $k-\varepsilon$ ) did not correctly predict the aerodynamic performance of the turbine due to the inaccurate simulation of the separated flow. Yao et al. [22] performed two dimensional simulations of a vertical axis wind turbine. The commercial CFD software FLUENT was used and two different turbulence models were tested: standard $k-\varepsilon$ and $k-\varepsilon$ RNG. Several differences were observed between the two models especially in the prediction of the total torque. Less difference was observed in the instantaneous velocity and pressure fields. McNaughton et al. [23] also performed 2D simulations of a vertical axis tidal current turbine using the open source code Saturne. Two turbulence models were used: The $k-\omega$ SST and the $k-\omega$ LRE (Low Reynolds number Effects) since they are more suitable to predict laminar separation presented in the dynamic stall phenomena. Numerical results show that the $k-\omega$ SST model overpredicts the maximum value of the power coefficient in comparison with the experimental result due to the dissipative effects of this model that is calibrated for fully turbulent flows. The $k-\omega$ LRE model does a better job in the prediction 
of the maximum power coefficient and the description of the separated flow in dynamic stall condition; nevertheless the minimum value of the power coefficient is highly under-predicted in comparison to the experimental data. Ghasemian et al. [24] performed an extensive review of CFD simulation techniques for vertical axis wind turbines. It was found that the $k-\omega$ SST transitional turbulence model achieve more accurate numerical results in comparison to any other fully turbulence model reported in the literature. The main reason for this finding is that the transitional models are capable of better predict the flow separation phenomena presented in these turbines. It was also found that turbulence models such as LES and hybrid RANS-LES are more accurate and better resolve the flow field but are still computationally expensive. Marsh et al. [25] performed two and three-dimensional simulations of a vertical axis tidal turbine using the commercial CFD software CFX and three different RANS models: Baseline-Reynolds Stress Model (BSL-RSM), $k-\omega$ SST and $k-\omega$ SST with transition. The best numerical results were found for 3D simulations with the $k-\omega$ SST transition model while the BSL-RSM and the $k-\omega$ SST with wall functions poorly predict the output power of the turbine. In general, 2D models did not correctly predict the turbine performance while 3D simulations using $k-\omega$ SST with wall functions did not achieve accurate results since it does not correctly predict flow separation at low rotational speeds. Even though, 3D models with $k-\omega$ SST transition were the most accurate, the computational cost is high (five times higher that $k-\omega \mathrm{SST}$ ) due to the large number of elements required in the simulation in order to completely resolve the boundary layer.

Another type of simulations of the flow around Vertical Axis Turbines is that oriented to obtain the influence of the turbine on the flow. In that case, the turbine and the blades constitute a momentum source for the flow which can be modelled by an actuator-type technique. In that sense, some recent works used Large Eddy Simulation coupled with an actuator-swept surface model [26] or an actuator line model [26-28] to simulate the flow through a VAWT focusing in the middle and far wake development. Such computations showed good comparison versus experimental measurements and constitute appropriate techniques to be applied for wind farm design and evaluation.

On the other hand, the aerodynamics of finite wings shows that the blade aspect ratio (AR) determines the lift to drag ratio. Smaller aspect ratio reduces the tangential forces and impacts negatively the power output. In that respect, the lift on the blade is directly proportional to AR but the induced drag is inversely proportional to it. Additionally, the detached vortices from the blade tip are related to the performance reduction of the machine, induce vibrations and increase the fatigue loads. These effects can be partially palliated using tip devices, which have the purpose of reducing drag but at the expense of rising the bending moment. Their main action is to weaken the intensity of the detached vortices at the blade tip and optimize the span-wise lift distribution. The tip devices increase the effective aspect ratio of a blade without materially increasing its span. Consequently, they are appropriated solutions for increasing the aerodynamic performance of a blade.

Several investigations have dealt with the influence of blade tip devices in horizontal axis wind turbines (e.g., [29,30]), both computationally and experimentally, which are discussed in [31]. As a main result Johansen and Sørensen [29] found that mechanical power and thrust of the turbine increased when winglet curvature radius decreased and when winglet height increased. Zhu et al. [32] carried out a numerical study about the influence of different design of winglets on the performance of a horizontal axis turbine; these authors considered both, asymmetric designs, with winglet tip facing the pressure or the suction sides of the blade, and a symmetric winglet design with two tips facing both sides of the blade all with the same cant and sweep angles. As a result of their steady state simulations, the symmetric winglet performed better than the asymmetric designs being able to provide an improving of power coefficient up to $5 \%$ regarding the original blade without tip devices.

On the other hand, for vertical axis wind turbines, although suggested since some years ago [33] there are not many studies. Sekiya et al. [34] studied experimentally the effect of a special elliptic winglets added to straight blade Darrieus wind turbine (so-called Bellshion blade) concluding that, for the studied range of velocities, the effect of the winglets was marginal. Ahmed and Netto [35], from experimental measurements in a wind tunnel, also reported that winglets were able to increase 
in more than $10 \%$ the performance of a vertical Darrieus wind turbine. On the other hand, in a CFD computational study, Amato et al. [36] compared the effect of endplates (called bulkhead shape), elliptical blade termination and three different blended types of winglets on the performance of a single blade Darrieus VAWT. These authors conclude that the adoption of the aerodynamic bulkhead increases strongly the performance while the role of winglets had to be more carefully studied as in two of them they estimated an increase of around $10 \%$ in performance respect to the original rotor without tip devices, but when the third winglet was evaluated, rotor efficiency was even reduced below the original one.

Reported evaluations of blade tip devices in vertical axis tidal turbines are scarce. In this respect, $\mathrm{Li}$ and Calisal [37] showed experimentally that the addition of endplates at blade tips improves efficiency of the turbine. However, to the best of knowledge of the present authors, no contributions are available in the literature dealing with the CFD evaluation of winglets on straight blade CFWT performance.

The present contribution performs 3D unsteady numerical simulations of the flow around a straight bladed CFWT using Computational Fluid Dynamics, considering the underlying turbulence of the flow and also viscous effects. The performance of three blade configurations is evaluated: a base case of standard straight blade and the same blade adding an asymmetric blade tip (blended winglet type) or a symmetric blade tip (hybrid fence-raked winglet type). In the simulations the sliding mesh method is employed, as it is the appropriated approach for describing the physical unsteady behavior of the flow around the CFWT blades. All the simulations in this study have been performed with the commercial software ANSYS-Fluent.

\section{Turbine Configurations}

The straight bladed CFWT studied in [14] has been chosen in this work as the basic case because all the geometric data were easily available. The turbine has a diameter $D=900 \mathrm{~mm}$, a span $H=700 \mathrm{~mm}$, with a resulting reference area $S_{\text {ref }}=D H=0.63 \mathrm{~m}^{2}$. The three straight blades are manufactured based on the symmetric NACA0025 profile. From the experimental cases, the so called 3S2R1 was selected; in that case the chord length is $c=132.75 \mathrm{~mm}$ giving a solidity $\sigma=0.89(\sigma=2 \mathrm{Nc} / D$ where $N$ is the number of blades). The rotational speed of the turbine is $\omega=60 \mathrm{rpm}=6.28 \mathrm{rad} / \mathrm{s}$.

The geometrical model simulated by CFD is a three-dimensional model of a real turbine. Additionally, as the main objective of this article is focused on the hydrodynamic performance of different blade configurations, neither supporting arms or shaft have been included in the simulation [14]. The computational domain consists of a rectangular box with an inner rotating ring subdomain in which the blades are located (Figure 1). To reduce the computational requirements the mid span was specified as a symmetry plane. The dimensions of the rectangular computational domain are $20 D$ in length $(x), 13 D$ in width $(y)$ and $3.5 D$ in height $(z)$, being $D$ the rotor diameter. This choice results in a blockage ratio of less than $1 \%$ which implies that the simulated configuration can be considered as nearly free stream conditions. Finally, the width of the ring subdomain is $20 \%$ of the turbine diameter.

The applied boundary conditions can be observed in Figure 1. In the CFD convention, a velocity inlet is defined on the west face while the right side is established as a pressure outlet; the lateral sides, north and south are specified as non-slip walls as well as the top face; finally the rotor mid-span was imposed to be a symmetry plane. As shown in Figure 1 bottom, the blades are inside the rotating sub-domain which is connected with the two steady domains (inner and outer) by means of interfaces defined by a sliding mesh boundary condition. Finally, as there was not experimental information about the turbulence level at the inlet, a value of $10 \%$ was tentatively assigned to that variable. At this point, it should be mentioned that this was the turbulence intensity at the inlet but, when the flow develops approaching the turbine, turbulence intensity values are lower. The influence of inlet turbulence intensity on turbine performance is addressed in Section 4.2. 


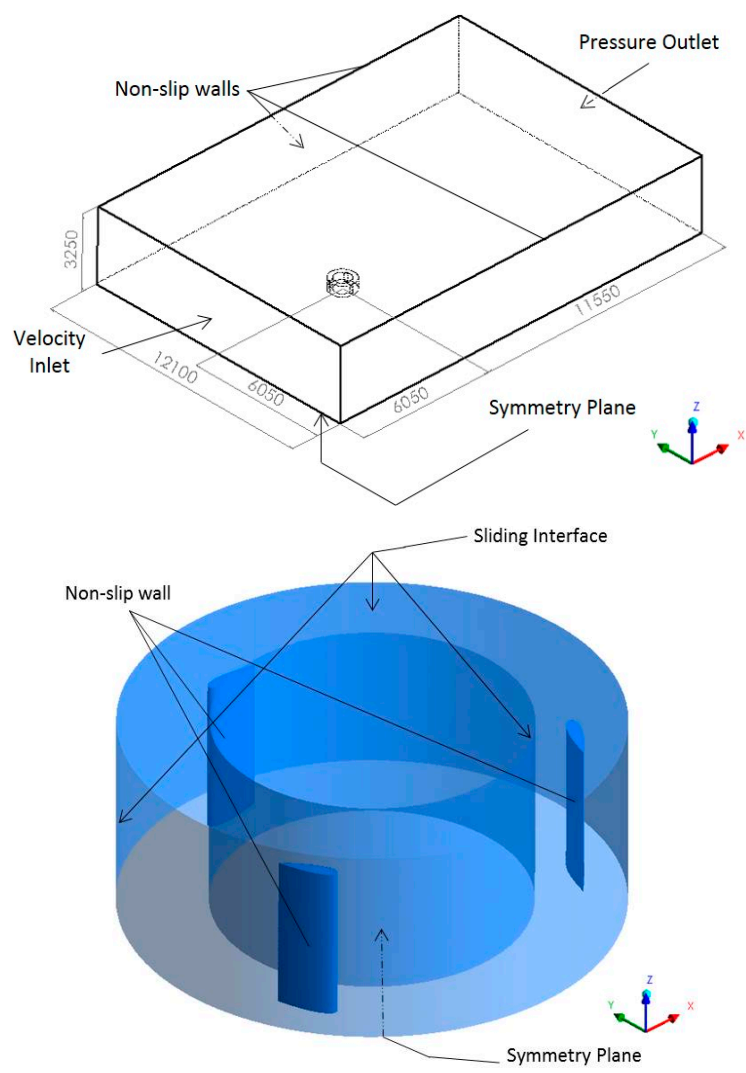

Figure 1. Sketch of the geometrical model of the turbine. (Top) perspective of the complete computational domain (dimensions are in $\mathrm{mm}$ ). (Bottom) rotating domain containing the blades.

In this study the following three blade configurations have been evaluated: the standard straight type and two additional ones that add winglets to the blade tips, one asymmetric and one symmetric (see Figure 2).

The asymmetric tip device (Figure 2 left) is directed towards the outer part of the rotating ring and it is inspired in the blended type of winglets [38]. The definitions of the different parameters describing the winglet are referred to Figure 1 of [29]. In this case, the cant and sweep angles were both chosen as $45^{\circ}$; the profile's chord at winglet tip is $0.5 c$, while the twist and toe angle are taken as zero. The winglet height is $5 \%$ of the total blade span. A similar configuration of winglet for a Horizontal Axis Wind Turbine blade was studied by Gupta and Amano [39], where it was demonstrated that a $45^{\circ}$ cant angle performed better than $90^{\circ}$. The join between the winglet and the blade has been left sharp at this moment. Although such sharp junction is known to produce interference drag in flying wings, this choice eases the generation of the geometry as well as the mesh. As the present work is intended to demonstrate the beneficial effects of such tip devices for the turbine performance regarding the standard straight blade base case, the sharp junction has been kept in this work.

The symmetric tip device is of hybrid fence-raked type (Figure 2 right) and it was inspired by $[40,41]$. The cant angle was chosen as $45^{\circ}$ and the sweep angle as $60^{\circ}$; the profile's chord at winglet tip is $0.25 c$, while the twist and toe angle are taken as zero. The winglet height is $7 \%$ of the total blade span. In this case, a similar winglet configuration in HAWT blade was considered in [32], although the height was somewhat shorter, and for a wing by [42]. As in the asymmetric winglet, the connection with the blade is sharp. 

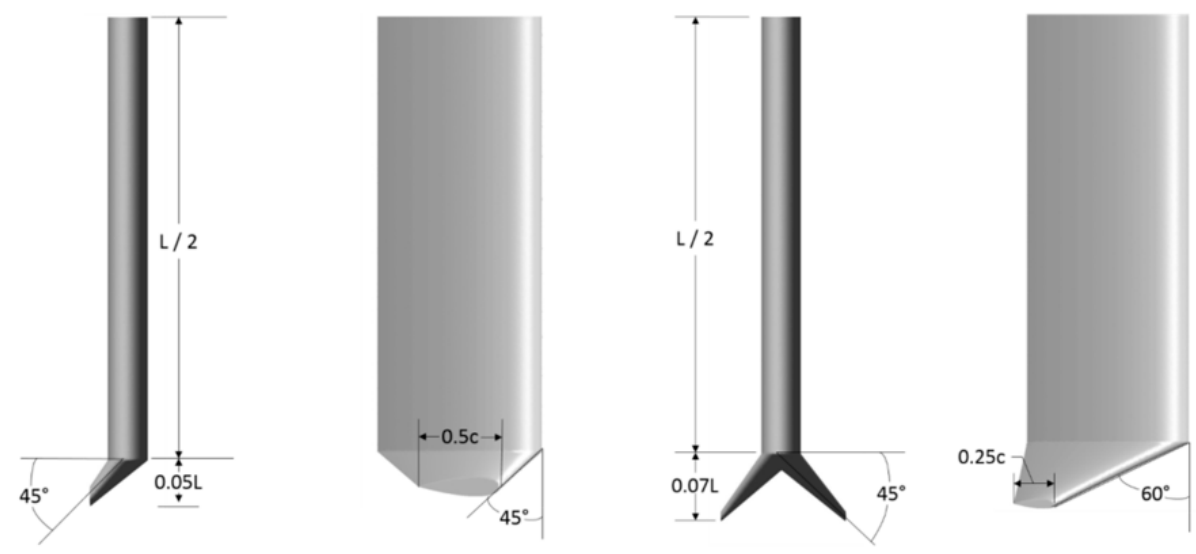

Figure 2. Geometrical details of the winglet design. (left) asymmetric winglet. (right) symmetric winglet.

The software ANSYS ICEM-CFD was employed to generate a non-structured mesh on the domain. As the interaction between fluid and the turbine happens on the blades surface, the mesh closest to them must be sufficiently fine in order to describe the boundary layer development with enough precision. Therefore, an O-grid topology mesh was employed comprising eleven layers of prisms. Beyond this region, the grid was based on tetras and, for ensuring a smooth transition, the aspect ratio of them was kept similar to that of the prisms. Further, also the grid density was higher in the turbine wake than upstream the CFWT with the objective of capturing the main characteristics of the flow in that zone. In the configurations with winglets and due to their geometrical complexity, special care was taken in the meshing process in order to get a good enough mesh quality. Figure 3 shows a close-up view of the employed surface mesh in the blade tip devices. As it can be seen, it is fairly uniform. The checked mesh quality parameters (available in ICEM-CFD) for the employed meshes in the three blade configurations are listed in Table 1.
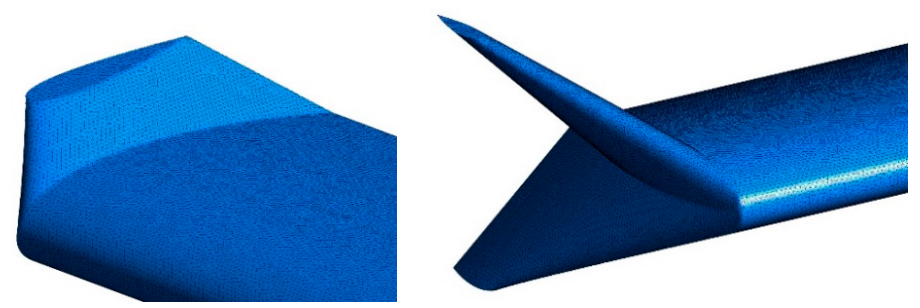

Figure 3. Details of the surface mesh generated in the blade tip devices: (left) asymmetric and (right) symmetric.

Table 1. Quality mesh parameters for the different blade configurations.

\begin{tabular}{cccc}
\hline Parameter & Straight Blade & Asymmetric Winglet & Symmetric Winglet \\
\hline Aspect ratio (average) & 0.86 & 0.83 & 0.83 \\
Equiangle skewness (average) & 0.35 & 0.38 & 0.39 \\
Determinant (average) & 0.95 & 0.93 & 0.92 \\
Expansion factor & 1.15 & 1.15 & 1.15 \\
\hline
\end{tabular}

\section{Simulation Setup}

The unsteady flow around the CFWT was described by means of the transient rotor-stator approach, using the sliding mesh technique. In such model, the turbine blades rotate with constant angular velocity respect to the steady domain. The position of the interface between both domains is updated at each time step guaranteeing a conservative interchange of fluxes between them. 
To model the turbulent behavior of the flow, the Transition Shear Stress Transport (Transition SST) turbulence model was employed. As stated in [43,44], it combines the $k-\omega$ and $k-\varepsilon$ models: $k-\omega$ model is used near of the wall and by a blending function it switches to $k-\varepsilon$ model far enough of the wall. In flows with strong adverse pressure gradient, such as those occurring around a CFWT, this model performs better than other two-equation models and it is the preferred option to describe the turbulent flow around rotating machines. The reason for using a transition turbulence model is that physically the airfoil Reynolds number is changing in time, so that several phenomena are actually happening in the boundary layer such as laminar boundary layer, transition to turbulence, laminar separation, flow reattachment and turbulent separation. Typically, a fully turbulence model cannot predict or capture these phenomena, even though the production of turbulent kinetic energy can be controlled with a damping function. In the case of the SST transition model, two extra equations control the laminar-turbulence transition: one for the intermittency (fraction of time that flow is turbulent in the boundary layer) and the other for the transition momentum thickness Reynolds number [43].

The inlet boundary conditions for turbulence in the SST transition model are specified by three parameters: intermittency $(\gamma)$, turbulence intensity $(T u)$ and turbulent viscosity ratio $(T V R)$. Based on $T u$ and TVR the values of turbulence kinetic energy $(k)$, turbulent frequency or specific dissipation rate $(\omega)$ and transition momentum-thickness Reynolds number $\left(R e_{\theta t}\right)$ are computed using the following expressions:

$$
\begin{gathered}
k=\frac{3}{2}\left(\frac{T u \cdot U_{\infty}}{100}\right)^{2} \\
\omega=\rho \frac{k}{\mu \cdot T V R} \\
R e_{\theta t}=331.50(T u-0.5658)^{-0.671}
\end{gathered}
$$

Equations (1) and (2) are based on isotropic turbulence while Equation (3) is an empirical correlation obtained by Langtry and Menter and implemented in Ansys Fluent [43]. In the range in which $T u$ was varied (see Section 4.2) the inlet turbulent variables adopted the values shown in Table 2. The value $\gamma=1$ indicates that the flow is assumed turbulent at the inlet.

Table 2. Turbulent variables values at the inlet for the SST transition model for the tested Tu levels.

\begin{tabular}{cccccc}
\hline$T u(\%)$ & $\gamma$ & $T V R$ & $k\left(\mathbf{m}^{2} / \mathbf{s}^{\mathbf{2}}\right)$ & $\boldsymbol{\omega}(\mathbf{1} / \mathbf{s})$ & $\boldsymbol{R} \boldsymbol{e}_{\boldsymbol{\theta} \boldsymbol{t}}$ \\
\hline 1 & 1 & 10 & 0.0004 & 39.3 & 580.2 \\
10 & 10 & 10 & 0.04 & 3992 & 73.5 \\
20 & 20 & 10 & 0.157 & 15,715 & 45.3 \\
\hline
\end{tabular}

In the computations, second order schemes are employed in the discretization of all differential equations, centered for the diffusion term and upwind for the advection term. The coupling of pressure and velocity is handled with the transient Semi-Implicit Method for Pressure-Linked Equations (SIMPLE) scheme. Temporal discretization was carried out by an implicit scheme of second order with a time step corresponding to $0.9^{\circ}$ degrees of turbine rotation; finally, a maximum number of 100 iterations is specified at each time step, employing a residual convergence criterion of $10^{-4}$.

The computational strategy for obtaining a converged solution is as follows: first, the steady flow around a fixed position of the blades is calculated, which acts as starting point for the unsteady computation; then, the transient simulation is run using first order schemes for a few turns (typically three or four) as an initial phase of flow development; when the power coefficient versus time has settled down showing a quasi-periodic curve, the discretization schemes are switched to second order; finally, the computation is continued for several turbine revolutions up to the averaged torque in a turn must differ less than $0.1 \%$ respect to the same value obtained in the previous revolution. The total number of turns needed to reach such convergence criterion depends of tip sped ratio; however, in the present study all the computations have been run for a real time equivalent to 20 turbine revolutions. 
A grid independence study was carried out to verify the numerical solution of the flow around the turbine. The selected objective variable was the torque coefficient, $C_{m}$, in the configuration of the straight blade. Therefore, following the standard process, three grids with different number of elements have been taken into account: a coarse mesh with around $2 \times 10^{6}$ elements (average $y^{+} 1.8$ ), a medium grid with about $4.3 \times 10^{6}$ elements (average $y^{+} 0.8$ ) and a refined grid with approximately $11 \times 10^{6}$ elements (average $y^{+} 0.5$ ). The difference in torque coefficient between the medium and the refined grid was around 3\%. Figure 4 shows the results of this verification study. Consequently, in order to keep an affordable computational time the medium grid was finally adopted.

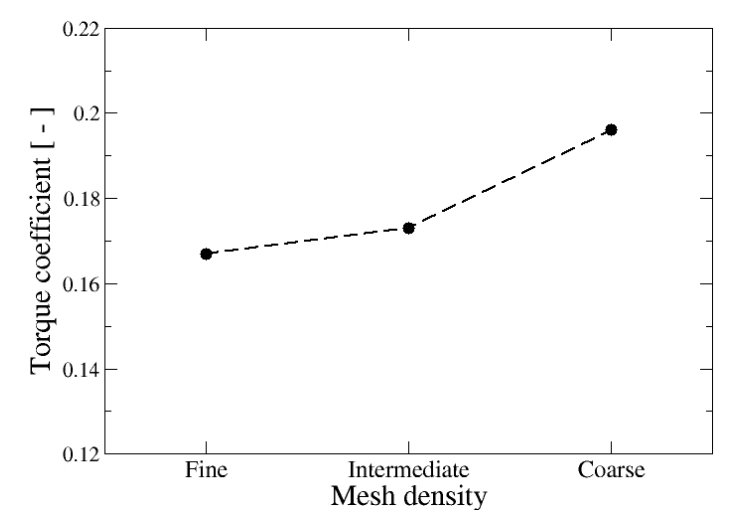

Figure 4. Average torque coefficient obtained in each grid considered in the grid independency study. Difference of torque coefficient between intermediate and refined grids is about $3 \%$.

\section{Results and Discussion}

Numerical simulation of the three turbine configurations, straight and winglet blades, was performed in meshes consisting of 4-5 million elements, according to the verification process mentioned above. As already mentioned in previous sections, a prism layer was built around the blades surface to ensure a smooth transition in size and skewness of the elements. The values of $y^{+}$in the computations are kept below 1 as required by the selected turbulence model, which also guarantees an adequate resolution of the boundary layer development around the blades [16]. This fact, together with the employed second order schemes in the spatial and temporal discretization, gives confidence about the obtained numerical results. The computed turbine operation point was that reported in [14], i.e., tip speed ratio $\lambda=1.745$ which is defined as $\lambda=\omega D / 2 V_{0}$, being $V_{0}$ the incoming water velocity. The performance of the turbine is given by the power coefficient defined by Equation (4):

$$
C_{p}=P / 0.5 \rho V_{0}^{3} S_{\text {ref }}
$$

where $P$ represents the CFWT power and $\rho$ the water density, $S_{\text {ref }}=D H$ is the turbine cross section being $H$ the blade span. Also important is the torque coefficient, which is defined as:

$$
C_{m}=M / 0.25 \rho V_{0}^{2} D S_{\text {ref }}
$$

which is related with the power coefficient through the tip speed ratio as: $C_{p}=C_{m} \lambda$. In (5) $M$ represents the torque that the fluid provides to the turbine.

The computational results are compared versus the experimental results of $[14,45]$ in the straight blade configuration and are presented in Figure 5. The measured values (Test legend) of torque coefficient were adjusted to compensate some extra friction that "was unaccounted for in the turbine test" [45] (Test adjusted legend), and both experimental points are shown in the graph. Moreover, the results obtained with the Double Multiple Streamtube (DMS) model and 2D CFD simulations by Dai et al. are included. Finally, the results of the three-dimensional computations performed in the present work are also presented in the same graphic. 


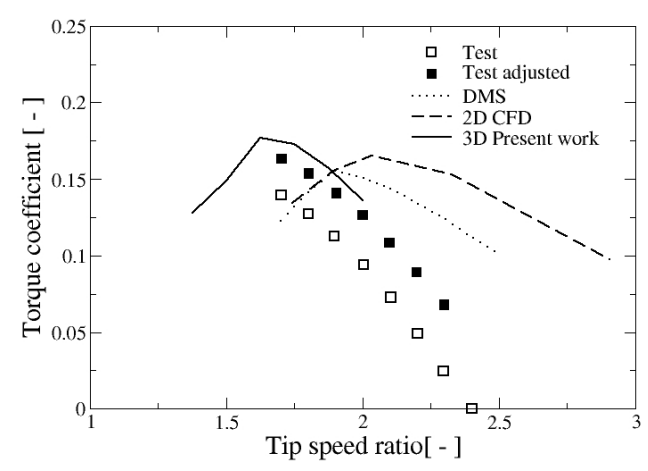

Figure 5. Average torque coefficient obtained in each grid considered in the grid independency study. Difference of torque coefficient between intermediate and refined grids is about $3 \%$.

As it can be readily seen from Figure 5, the DMS and 2D CFD computations overestimate the tip speed ratio in which the maximum $C_{m}$ is reached. Also, the trend of the curve shown by these computations differs from that of the experimental measurements, providing substantially higher torque coefficient for TSRs larger than two. In the DMS model this attributed to the well-known deficiencies of such approach when dealing with high solidity turbines. On the contrary, the obtained $3 \mathrm{D}$ results provide values close to the experiments (adjusted test curve) showing also the same curve tendency. However, the simulations overpredict slightly the measurements. This behavior was expected as the numerical model only considers the blades not including the shaft and supporting arms, which are known to decrease the turbine efficiency. As a result, the present numerical computations are close enough to the experimental measurements, reproducing also the shape of the curve, and improve greatly the simplified models predictions. Therefore, the present computational simulations can be considered validated in the straight blade configuration.

The influence of adding winglets at the blade's tip on turbine performance is analyzed in the next section.

\subsection{Analysis of Influence of Winglets in Turbine Performance}

After a few turns, around $10, C_{m}$ reached a quasi-periodic regime (with second order schemes) after initial transient. However, in this study the total number of performed turns has been 20 to assure that the averaged torque in a revolution differs less than $0.1 \%$ regarding the value obtained in the previous revolution [46].

As the turbine rotates, the incoming velocity approaches the blades with varying angles of attack resulting, for certain angular positions, in a separation of the boundary layer and detachment of vortices. Additionally, the flow downstream the turbine has lower speed than upstream, which is reflected in a smaller contribution to torque (Figure 6). As a consequence, in a revolution, a single blade generates positive contributions for $C_{m}$ but in some angle ranges, the produced $C_{m}$ can be slightly negative. Figure 6 (top) shows the evolution of the torque in a turn for the first blade. At azimuthal angle $\theta=0^{\circ}$, the blade chord is parallel to the incoming flow (i.e., with angle of attack $\alpha$ of zero degrees); at this position $C_{m}$ starts slightly negative, experiencing a minimum value around $25^{\circ}$; then the curve increases monotonically until reaching the absolute maximum at roughly $100^{\circ}$; after that, $C_{m}$ decreases up to $180^{\circ}$ where it has a local minimum, slightly higher than the absolute one, for increasing again attaining a secondary maximum, slightly positive, at about $220^{\circ}$; finally, the torque coefficient decreases gently up to finishing the turn where it becomes hardly negative again. The total torque generated by all the blades yields a quasi-periodic curve which presents three maxima and three minima in a revolution (Figure 6 bottom). Such number is equal to the turbine number of blades, three in this study. From this last figure, it can be clearly seen that both maxima and minima of the total $C_{m}$ have higher absolute value than the $C_{m}$ of only one blade, due to the different contributions of each blade to torque at those angular positions. In the studied case there is an interaction among the 
blades that adds the primary and secondary maxima of consecutive blades, resulting in a noticeably higher $C_{m}$ than that produced by one blade.
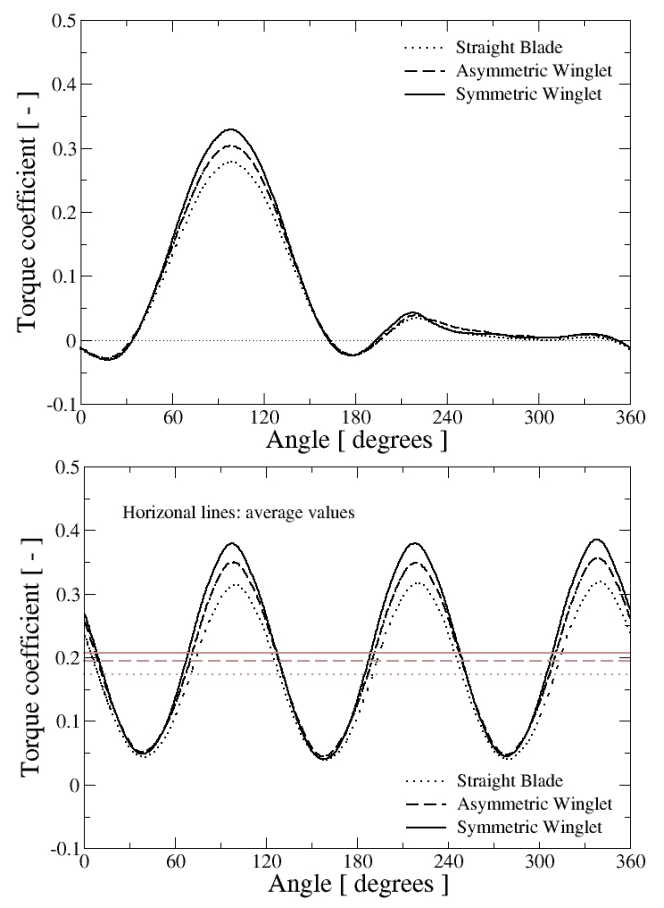

Figure 6. Torque coefficient evolution along a turn at $\lambda=1.745$ for the three studied configurations. Single blade (top) and three blades (bottom).

The $C_{m}$ values obtained for the three winglet configurations can be also appreciated in Figure 6 . Light color lines indicate the average values of torque coefficient along this revolution.

The straight blades base case presents the lowest maxima and minima, which reflects in the lowest turbine performance. Both winglet cases provide slightly higher minima and substantially higher maxima than the base case, resulting in a noticeable higher efficiency. From them, the symmetric hybrid fence-raked tip device provides the highest maxima and mean values for the power coefficient. Therefore, from Figure 6 is clearly seen that the improvement in efficiency generated by the blade tip devices is mainly due to the enhancement of the maximum power delivery at the angular positions where the lift attains a maximum. According to [8], is precisely in such locations where the trailing tip vortex is close to its maximum strength; nevertheless, the winglets are specifically designed to weaken such vortex, consequently implying lower drag and higher torque delivered by the fluid to the turbine.

From the performance results shown in Figure 6, it is obvious that the symmetric winglets results predict a remarkable improvement for $C_{m}$ which translates in an increase in turbine performance of around $20 \%$, regarding the standard straight blades configuration. These results agree with previous studies $[29,35,36]$.

Another interesting parameter of a CFWT is the thrust coefficient, i.e., the non-dimensional parameter associated to the force experienced by the turbine in the direction parallel to flow. The obtained results for such coefficient are shown in Figure 7. The top figure shows the evolution of the thrust coefficient of a single blade along a revolution, whereas the bottom plot illustrates the same variable for the complete CFWT. As it can be readily seen, the shape of the curves in Figure 7 (top) is pretty similar to those of the torque coefficient. At $\theta=0^{\circ}$ the thrust coefficient is very small as in this position the flow is parallel to the chord; increasing the azimuthal angle it increases up to $\theta=90^{\circ}$ where the blade is located exactly perpendicular to the incoming velocity. The thrust decreases then up to values close to zero in the azimuthal position of $180^{\circ}$, where the chord is again parallel to the flow. In the downstream semicircle, the thrust on the blade increases again up to $\theta=225^{\circ}$ and 
later it decreases gently up to $360^{\circ}$ reaching again its minimum value. Due to the perturbed velocity field in the downstream semicircle, the absolute value of the thrust coefficient here is much lower than in the upstream semicircle. The total thrust coefficient on the three blades is presented in Figure 7 (bottom). Curves present three maxima, corresponding to the positions where any of the blades is orthogonal to the incoming flow in the upstream semicircle (i.e., $\theta=90^{\circ}, 210^{\circ}$ and $330^{\circ}$ ) and three minima placed at $\theta=30^{\circ}, 150^{\circ}$ and $270^{\circ}$, where the flow direction is perpendicular to any of the blades in downstream semicircle.
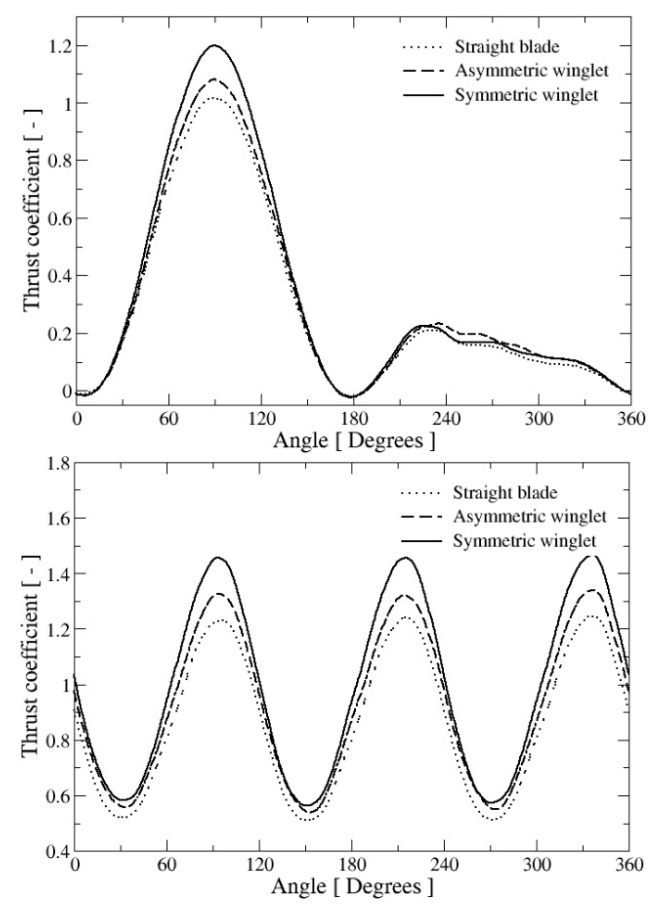

Figure 7. Thrust coefficient of the turbine depending on the blade configuration. Single blade (top) and three blades (bottom).

The shape of the curves in Figure 7 is the same for the three blade configurations. However, the maximum of thrust coefficient for a single blade is the highest for the symmetric winglet blade and the lowest for the configuration without winglets, but of similar magnitude in the rest of the revolution. When the total thrust is taken into account, the symmetric winglet blade presents not only the highest maxima but also the highest minima, while the straight blade experiences the lowest maxima and minima among the three configurations. Therefore, it is concluded in our case that the addition of winglets to the straight blade not only increases performance but also the thrust coefficient, a fact that must be accounted for during the blade design process.

The total fluid-dynamic force $\mathrm{F}$ acting on the blades can be projected on the directions parallel (tangential component, $F_{t}$ ) and perpendicular (normal component, $F_{n}$ ) to the profile chord, as it is shown in Figure 8. The software computes such force in a $x-y$ coordinate system; therefore, force coefficients $\left(C_{x}\right.$ and $\left.C_{y}\right)$ are obtained in such a reference system and a coordinate transformation (rotation) must be performed to obtain the force coefficients in the $t-n$ reference system (tangential-normal) in terms of the angular position $\theta[1]$ :

$$
\begin{aligned}
& C_{t}=C_{x} \cos \theta+C_{y} \sin \theta \\
& C_{n}=C_{y} \cos \theta-C_{x} \sin \theta
\end{aligned}
$$

where $C_{t}$ is the non-dimensional value of the tangential force, which is responsible for the torque transmitted by the flow to the turbine. 


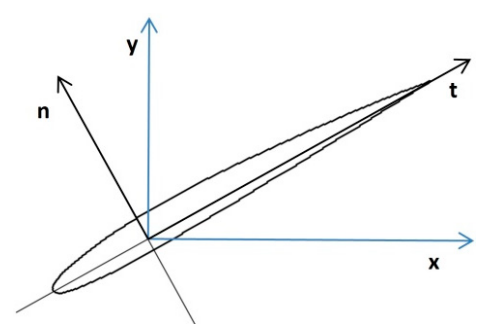

Figure 8. Coordinate systems implied in the definition of force coefficients. Flow is horizontal from left to right (positive $x$ direction) and turbine rotates counter-clockwise.

As it can be appreciated from Figure 9 top, negative values of tangential force are needed to obtain a positive torque provided by the blade. $C_{t}$ is negative in the upstream semicircle presenting an absolute minimum around $\theta=90^{\circ}$ and reaching a slightly positive maximum at $\theta=180^{\circ}$. In the downstream semicircle, the tangential coefficient crosses zero value at about $\theta=200^{\circ}$ to be kept gently negative up to $\theta=360^{\circ}$. The only angular positions where $C_{t}$ is positive are in a small range around $\theta=0^{\circ}$ and $180^{\circ}$, where the chord is parallel to the incoming flow.
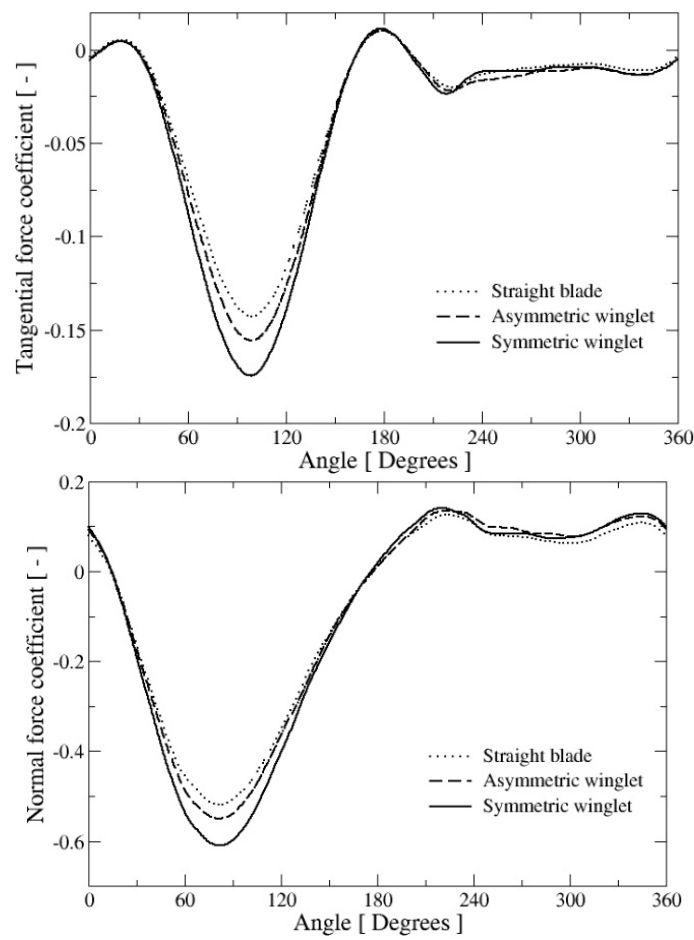

Figure 9. Tangential (top) and normal (bottom) force coefficients of a single blade depending on blade configuration.

$C_{n}$ is the non-dimensional value of the normal force, and it is connected with cycle loading and fatigue on the CFWT shaft. In the $t-n$ system (Figure 8 ) the normal force tends to be negative in the upstream and positive downstream semicircle, a fact that is corroborated by Figure 9 (bottom). At $0^{\circ}$ the normal force is positive, but around $15^{\circ}$ it becomes negative, reaching its minimum value approximately at $80^{\circ}$. Then the normal force coefficient increases and near $175^{\circ}$ becomes positive again, keeping a roughly constant value during the downstream semicircle.

In accordance with what was found in the analysis of the torque and thrust coefficient, the tangential and normal force coefficient are, in absolute values, the highest for the symmetric winglet blade configuration and the lowest for the straight blade, being the asymmetric case between them. 
In summary, in the present study the blade with winglets provides higher power coefficient estimation than for the straight blade configuration. However, this is not always the case: Amato et al. [32] found that it is also possible to obtain the contrary effect, i.e., a performance prediction lower than in the case of the straight blade. Nevertheless, interestingly, the study of Gupta and Amano [39] in HAWT's demonstrated that a winglet forming a cant angle of $45^{\circ}$ had better performance than the same winglet forming a cant angle of $90^{\circ}$ in agreement with the results presented in [29]. As in our study the cant angle of both winglet types is $45^{\circ}$, the present results agree with those obtained by [39].

\subsection{Sensitivity of Results versus Turbulence Modelling}

To study the sensitivity of results regarding the employed turbulence model, three additional turbulence models have been tested: RNG $k-\varepsilon$ [47], SST $k-\omega$ [48] and Reynolds Stress Model (RSM) [49].

The RNG $k-\varepsilon$ turbulence model extends the application of the standard $k-\varepsilon$ to flows with non-equilibrium strain rates and it has been previously applied in simulation of vertical axis wind turbines [8]. The SST $k-\omega$ turbulence model has been widely applied in turbomachinery problems where it has become a kind of standard, so it is a good choice for comparison. In contrast to the two-equation eddy viscosity models, the Reynolds Stress Model solves independent transport equations for each component of the Reynolds Stress Tensor, avoiding the Boussinesq approximation for closing them. It is preferred when the underlying turbulence of the flow field is anisotropic, there is strong curvature of the streamlines and/or the flow is characterized by strong swirl and adverse pressure gradients.

Results obtained with the four turbulence models in the three considered blade configurations can be appreciated in Figure 10. Although a dispersion of the predicted average torque coefficients can be noticed, all the turbulence models indicate that the blade performance is higher when winglets are added to the blade tip. These computations suggest that the blade equipped with the symmetric winglet is able to extract the highest power from the water current, followed by the asymmetric winglet, both being superior than the reference straight blade case. Therefore, the trend shown in Figure 6 is corroborated by the other turbulence models.

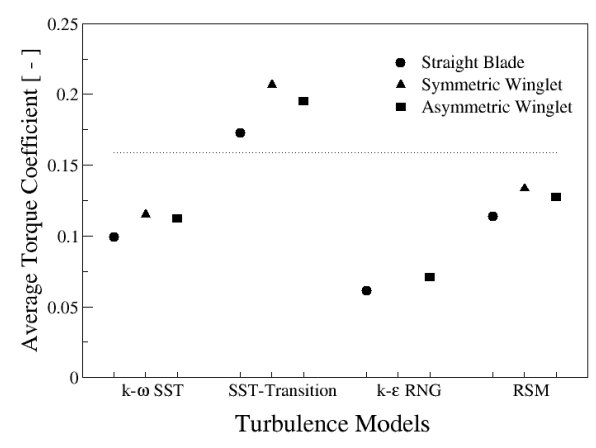

Figure 10. Sensitivity of average torque coefficient versus turbulence models. In all cases the symmetric winglet provides the maximum performance. The dashed line shows the experimentally expected value.

There is still one comment to be mentioned: the result corresponding to the symmetric winglet with the RNG $k-\varepsilon$ turbulence model is absent. The reason is that it was not possible to get convergence on this case using second order numerical schemes. When the numerical scheme was switched to second order, the dissipation equation always started to behave unstably leading to an eventual divergence.

In Figure 10, the dotted line shows the experimental value of [8]. From that figure, it can be seen that the closest values to the expected value are provided by the $k-\omega$ SST Transition model and the RSM model. The Transition $k-\omega$ SST model good prediction of the expected value can be explained by the fact that it is a low-Re turbulence model that does not rely on empirical damping functions 
(as typical two-equation models). The advantages of this model in the prediction of boundary layers with adverse pressure gradient and separation are well-known. On the other hand, the RSM model can provide a better performance than two equation turbulence models especially in flows with sudden changes in the strain rate and with high swirl and rotation, which is the case of the simulation of vertical axis turbines.

Moreover, the obtained three-dimensional results are in qualitative agreement with the two-dimensional computations of [1], where the RSM and Transition SST turbulence models provided the closest results to the experimental expected torque coefficient value. However, in the 3D case the predictions of the Transition SST model are above the standard SST while in the 2D computations the contrary happens. Marsh et al. [25], also found in their 3D computations that the transition version of the SST turbulence model provided higher values than the standard version for tip speed ratios higher than that corresponding to the maximum power coefficient, but the contrary happens for lower tip speed ratios. Additionally, the same trend is obtained in the case of own computations of a horizontal axis hydrokinetic turbine.

A fact that is not frequently studied in the CFD simulations of CFWT's is the effect of the inlet turbulence intensity $(\mathrm{Tu})$ on turbine performance, i.e., torque coefficient. Figure 11 shows such influence in the case of symmetric winglet blade with the SST Transition turbulence model for a single blade (top) and three blades (bottom). As it can be seen from Figure 11 (top) the maximum torque is slightly increased with turbulence intensity, but the local maximum, around $\theta=220^{\circ}$, noticeably decreases for higher turbulence levels. From about $230^{\circ}$ to $360^{\circ}$, in the downstream semicircle, apparently the torque is not affected by the turbulence intensity of the underlying flow. As the total torque is the sum of the values experienced by the three blades, it is found that the $C_{m}$ maxima decrease with turbulent intensity but the minima increase with it (see Figure 11 bottom). The net result is that the average torque in a revolution increases with the turbulence level at inlet; in the present case such increment is close to $6 \%$ when the inlet turbulent intensity increases from $1 \%$ to $20 \%$. Although not shown, the average thrust coefficient also increases slightly when turbulence is augmented ( $1 \%$ in this case). Therefore, such parameter should also be taken into account in the sensitivity analysis of the CFD computations of CFWT's.
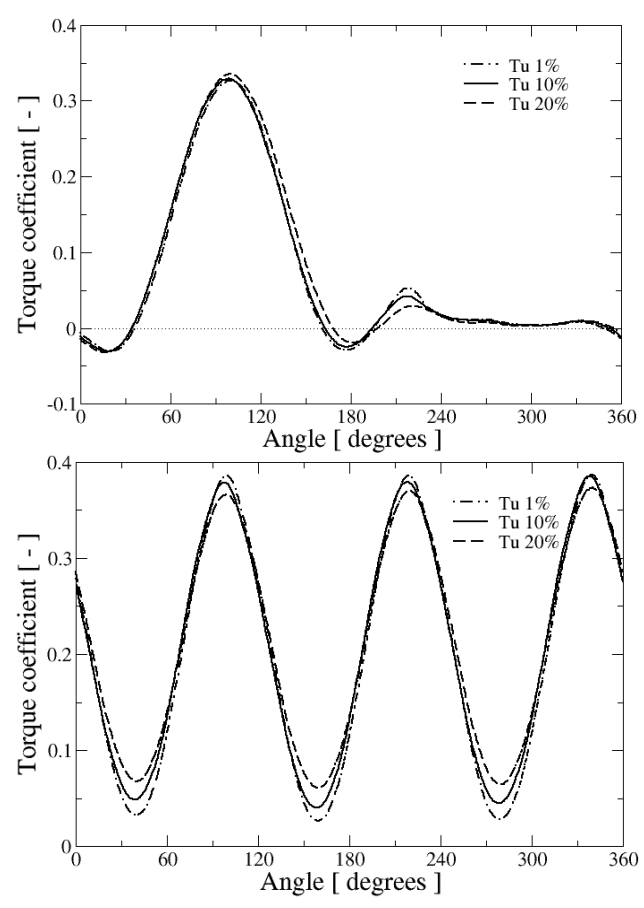

Figure 11. Torque coefficient evolution along a turn at $\lambda=1.745$ for the symmetric winglet configuration and three different turbulence levels $(\mathrm{Tu})$ at the inlet. Single blade (top) and three blades (bottom). 


\subsection{Visualisation of Flow Structures}

Because CFD provides the flow variables along the entire fluid domain, it is a tool that can be exploited to identify flow structures and study their behavior. In order to illustrate some of them generated in the flow around a CFWT, vorticity isosurfaces and skin friction line patterns on the blade surface will be shown in the following.

It is known that the skin friction lines along a surface allow the analysis of the flow separation near it [50]. In fact, it is an experimentally common observation that the convergence of oil streak lines into a particular line is a necessary condition for the occurrence of flow separation.

Singular points in the pattern of skin friction lines occur at isolated points where the wall shear stress is zero and largely determine its distribution on the surface. There are three types of singular points: nodal points, focus and saddle. A nodal point is a singular point common to an infinite number of skin friction lines with the same direction, either emerging from it or converging to it; a nodal point of attachment is typically a stagnation point on a forward facing surface (i.e., the nose of a body). In a focus, the skin friction lines spiral around the singular point, either away of it or into it. A saddle point is determined by the intersection of only two skin friction lines which converge on a direction and diverge in the other; all the other lines miss the singular point and take direction consistent with those of the adjacent particular lines [50]. In particular, a skin friction line going through a saddle point is called a separation line; if the adjacent lines converge towards it, it is called a detachment line (where the flow detaches from surface) and if adjacent lines diverge from it, it is called an attachment line (where the flow attaches to surface). The direction of the lines can be determined by observing the wall shear stress vector on the surface. In a skin friction line pattern on a surface the number of singular points must satisfy the topological rule that the number of nodes (nodal points plus foci) must exceed the number of saddle points by two. Typical configurations are the combination of nodal points with saddle points or the focus - saddle that generates a concentrated vortex filament.

Figure 12 (left) shows the distribution of the skin friction lines along blade intrados (side pointing toward rotation axis) of the three blade configurations at the azimuthal position $\theta=180^{\circ}$. In Figure 12 the flow is from left to right. As it can be readily seen, the three types of mentioned singular points are identifiable. As examples, some nodal (N), focus $(\mathrm{F})$ and saddle (S) points are marked in the figures. The separation line (red line AA in the plots) is a detachment line where the flow separates from the surface. This fact is clearly seen in the right column of Figure 12 that shows the $60 \mathrm{~Hz}$ vorticity isosurface around the blades: along the AA line the isosurface grows in the y direction illustrating the boundary layer detachment. It can also be seen by checking the shear stress vectors at the surface where the adjacent lines converge to AA (not shown). As a result, the skin friction lines pattern and the shape of the vorticity isosurface differs noticeably among the three blade configurations, especially near the blade tip: in the straight blade the typical long trailing vortex is developed extending downstream following a curved path; the asymmetric winglet is able to weak such a vortex, delaying its detachment; and, in the symmetric winglet case, also a detachment of the vorticity isosurface happens in the intrados of the winglet and no trailing vortex is observed. An analysis of the variation of the skin friction lines and non-dimensional form of the wall shear stresses along a revolution in the case of the symmetric winglet is discussed in Appendix A.

Additionally, when looking to the shape of the vorticity isosurfaces on the extrados (blade side pointing outward rotation axis) a rough, corrugated structure can be clearly distinguished (Figure 13 top). Here, only plots for the straight blade are shown as illustration, although the same is observed for the winglet cases. As far as the authors are aware such corrugated isosurface has not been reported before in the literature. On top of the wrinkled surface several stream-wise tubular structures are visible, inside of the blue ellipses, which we call "vorticity fingers". Such corrugated pattern follows the direction of the skin friction lines which are the footprint of counter-rotating quasi-stream vortices, similar to the coherent structures which appear in the near wall region of a turbulent channel flow. This can be demonstrated by coloring the $60 \mathrm{~Hz}$ vorticity isosurface with the stream-wise vorticity component ( $\mathrm{z}$ in this case): an alternation of blue and red colors identifies the counter-rotating vortices 
developing in the stream-wise direction (see Figure 13 bottom). Moreover, a span-wise vortex close to detachment can also be appreciated in its trailing edge in green color.

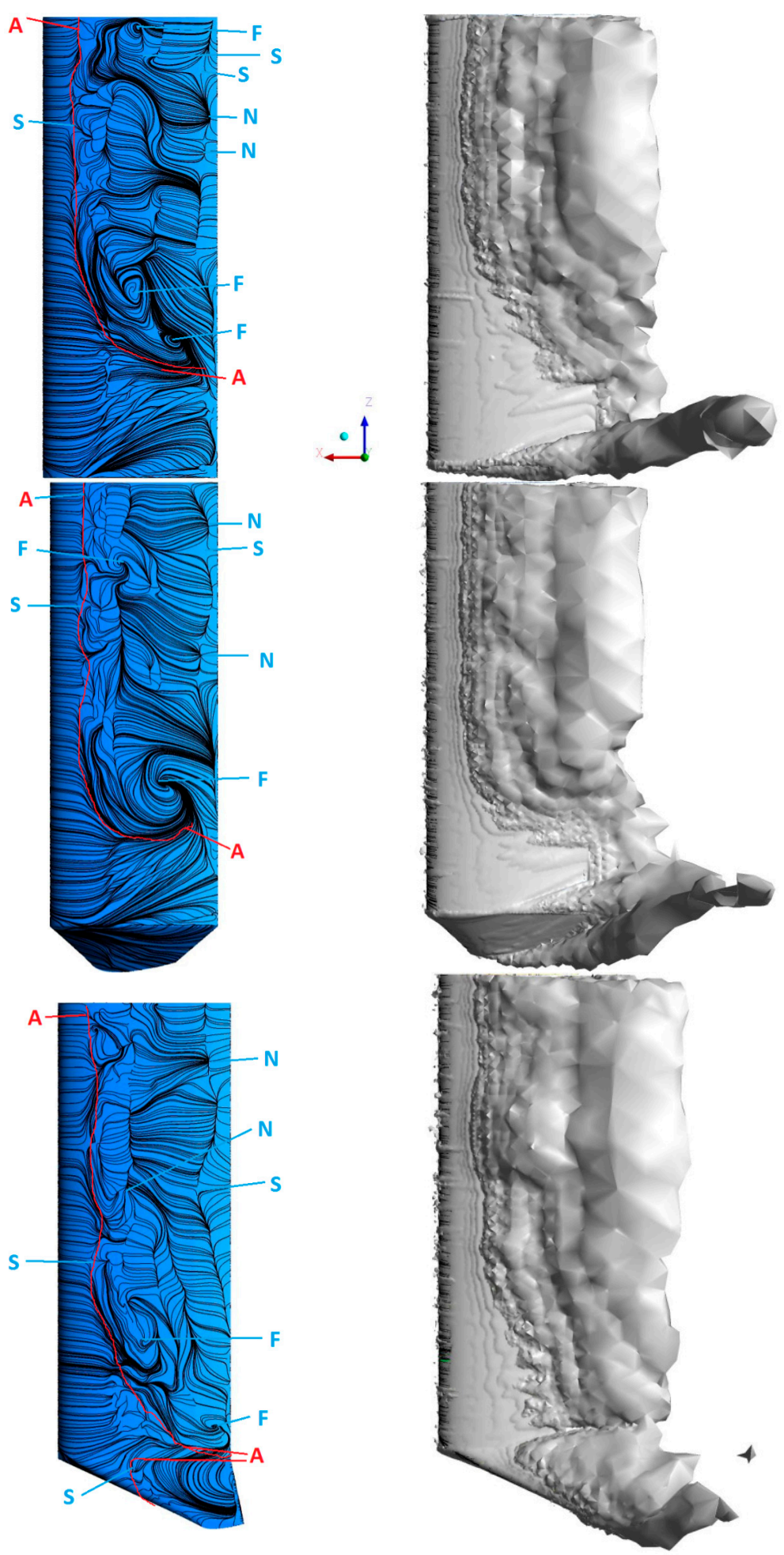

Figure 12. Skin friction lines (left) and $60 \mathrm{~Hz}$ vorticity isosurface (right) on the intrados surface at azimuthal position $\theta=180^{\circ}$. Upper row: straight blade; middle row: asymmetric winglet; bottom row: symmetric winglet. Only the lower half of the blades is shown. 
Finally, to illustrate the behavior of the transitional boundary layer along a blade revolution the intermittency field is discussed. Intermittency is defined as the fraction of time that the flow is turbulent in a point in the boundary layer. It adopts the value of 1 in fully turbulent flow and 0 in laminar flow. In the locations of the boundary layer where turbulent structures start to be visible, intermittency begins to deviate from zero [51]. Together with an empirical transport equation for the transition momentum-thickness Reynolds number, the transition features of boundary layer are modeled [43]. This fact is relevant for CFWTs as the values of skin friction coefficient along the blades depend on the boundary layer development which has an impact in their performance (see Figure 10).

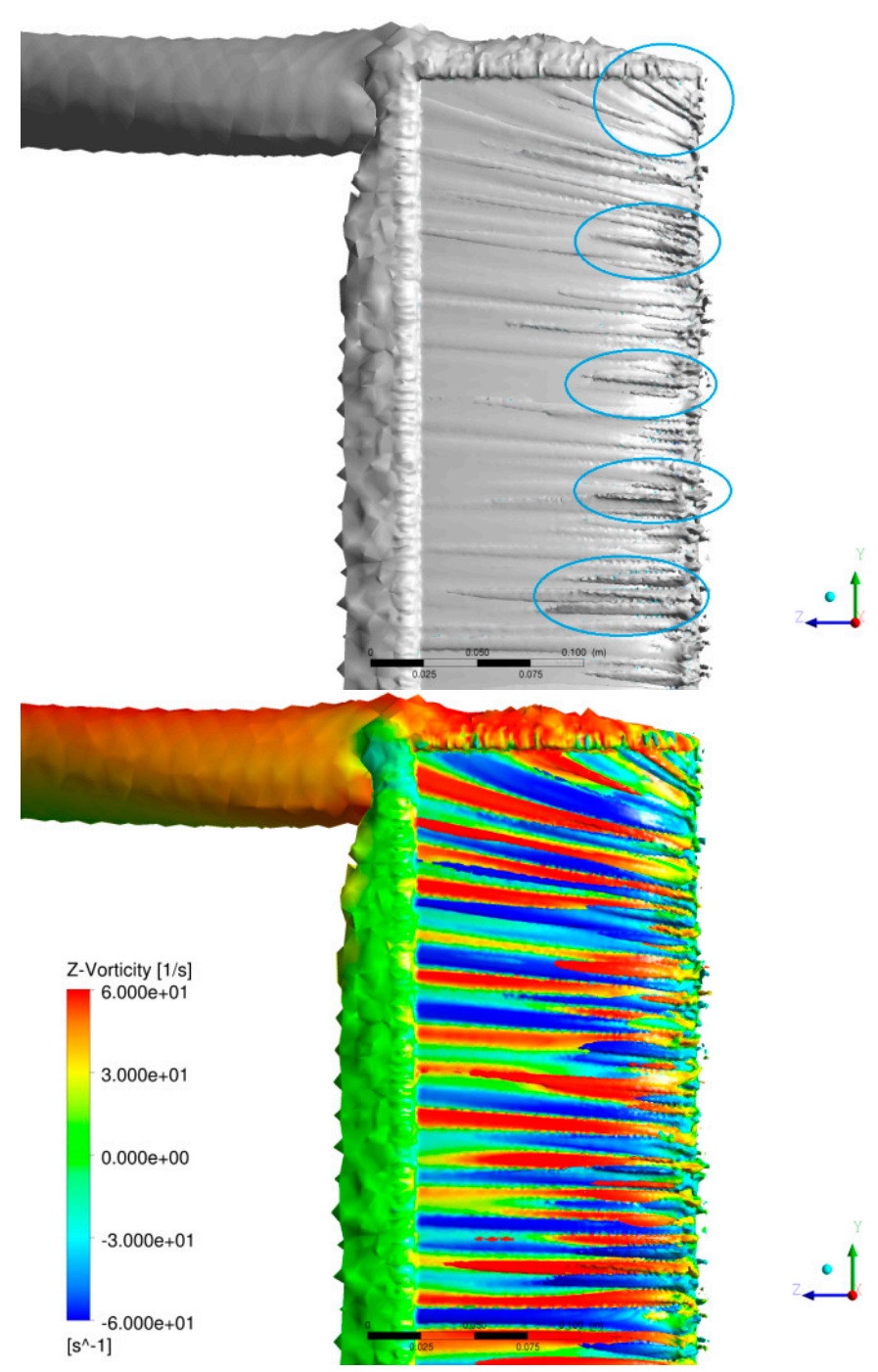

Figure 13. (Top) Isosurface of vorticity equal to $60 \mathrm{~Hz}$ on the extrados of the straight blade. (Bottom) same isosurface colored by values of streamwise vorticity ( $z$-component).

Figure 14a-d illustrate the evolution of the intermittency field during one cycle of the symmetric winglet turbine. The behavior of this variable is rarely shown in the literature, so it deserves some attention. The $2 \mathrm{D}$ view corresponds to a plane located at $5 \mathrm{~cm}$ from the symmetry plane and a value of 1 indicates always turbulent flow. In that figure, the water flow progresses from left to right and transition from one image to next correspond to a change of $30^{\circ}$ in the airfoil position. 


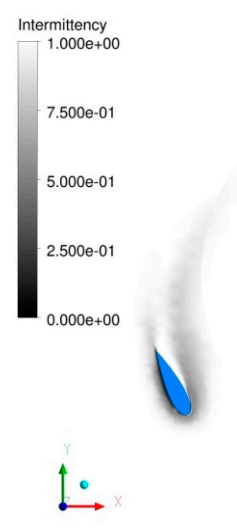

(a)

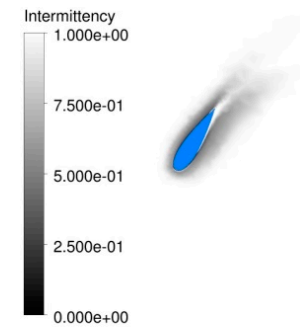

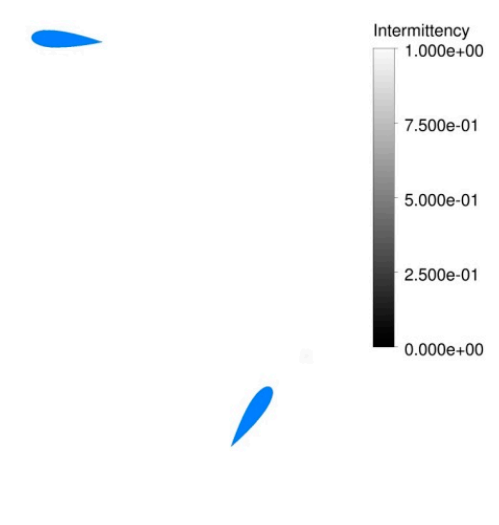

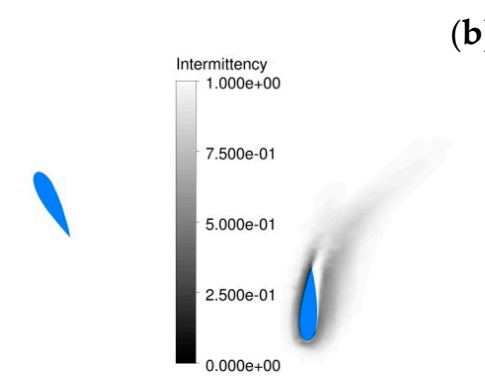

(b)

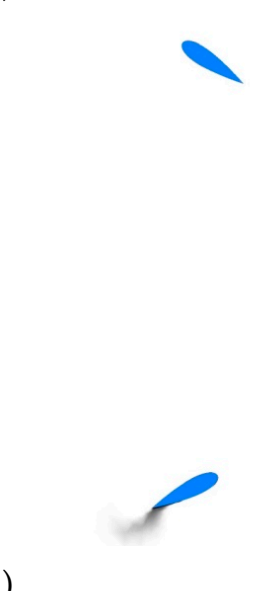

(d)

Figure 14. Intermittency field evolution in one cycle in a plane located $5 \mathrm{~cm}$ from the symmetry plane. From one image to the next, each airfoil experiences a change of $30^{\circ}$ in its angular position. First blade located at (a) $0^{\circ}$; (b) $30^{\circ}$; (c) $60^{\circ}$; (d) $90^{\circ}$.

When the cycle starts $\left(\theta=0^{\circ}\right)$, the boundary layer in both upper and lower sides of the blade has a noticeable portion of laminar boundary layer (see Figure 15a) very similar to the observations of [1]. In fact, in all the blade positions along the cycle it is possible to observe that there is an area with intermittency values noticeably lower than 1 around the profile. In the downstream semicircle (actually in the range of $210^{\circ}-30^{\circ}$ ) the extension of such area is circumscribed mainly to the boundary layer, which is illustrated in Figure $15 \mathrm{~b}$ for $\theta=270^{\circ}$. However, in the upstream semicircle (roughly between $30^{\circ}$ and $210^{\circ}$ ) the intermittency field presents values lower than 1 in wide regions around the profiles, reaching the wake (Figure 14a-d). In such regions the eddy viscosity and the production of turbulent kinetic energy is lower than in fully turbulent conditions. Therefore, the effects of laminar-turbulent boundary layer transition are present along the whole revolution of the turbine.

Figure $15 \mathrm{c}$ shows the isosurface of intermittency with value of 0.5 at the blade azimuthal position of $\theta=60^{\circ}$ for the symmetric winglet. As it can be readily noticed, the isosurface at the intrados presents also the corrugated structured inherited of the vorticity distribution and it does not reach the trailing edge, consistent with the contour plot of Figure 15c. On the other hand, such isosurface wraps around the extrados of the blade showing that in such instantaneous position the boundary layer development is entirely laminar. 


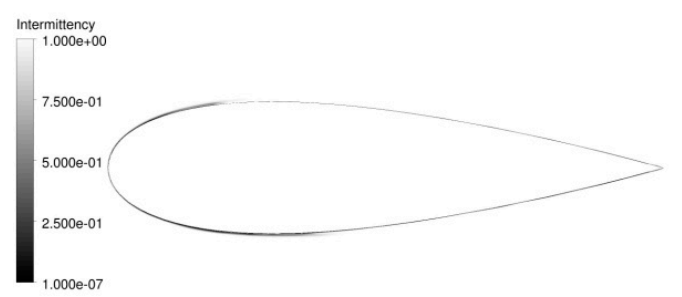

a)

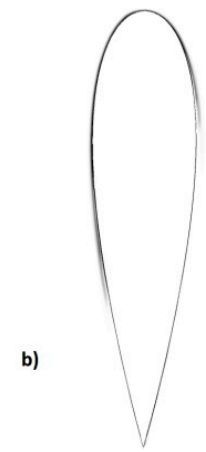

c)

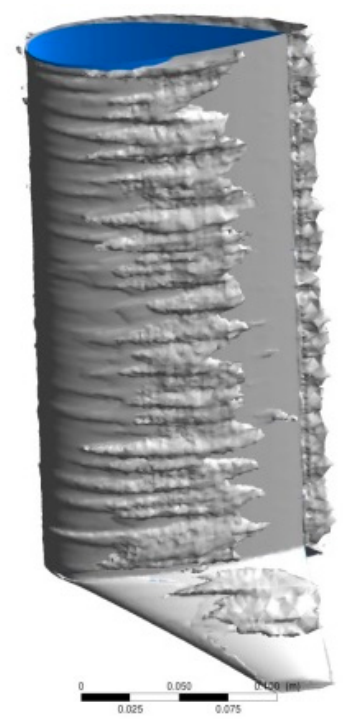

Figure 15. Close up of the intermittency contours at selected azimuthal locations: (a) $\theta=0^{\circ}$; (b) $\theta=270^{\circ}$; (c) isosurface of intermittency value equal to 0.5 at azimuthal position $\theta=60^{\circ}$ at the intrados (symmetric winglet blade).

\section{Conclusions}

The presented computational results show clearly that the performance of the CFWT can be improved by adding winglets to the blades' tips. The enhancement in turbine efficiency is mainly due to the weakening of the trailing tip vortex at locations of maximum lift in a blade turn. This fact implies the reduction of lift induced drag over the blade, because the tip devices increase its effective aspect ratio without significantly modifying blade's span. From the studied configurations, the maximum improvement predicted in the CFWT power coefficient is attained by the symmetric hybrid fence-raked winglet and it is estimated in a $20 \%$ regarding the straight blade base case. Moreover, the addition of winglets also increases the thrust on the CFWT, a fact that should be taken into account in the design process of the turbine. The obtained results are corroborated in a sensibility study considering various turbulence models, as the same trends are obtained with the four models considered in this work. Additionally, the effect of turbulent intensity of the incoming flow on turbine performance has been evaluated. It has been found that increasing the turbulence levels at the inlet, keeping them moderate (lower than $20 \%$ ), increases the global torque on the turbine as well as the thrust on it. Finally, visualization of skin friction lines pattern and their connection with vorticity isosurfaces, illustrating the flow detachment in the three blade configurations, has been carried out. It has been found that vorticity isosurfaces present a corrugated structure which is indicative of the presence of counter-rotating quasi-stream vortices, similar to the coherent structures which appear in the near wall region of a turbulent channel flow or in the development of the boundary layer along a flat plate. Moreover, a qualitative description of the behavior of the intermittency (variable not shown 
frequently in the literature) along one turbine revolution has been presented, demonstrating that laminar-turbulent boundary layer transition effects are important at all blade positions. Future work will be focused in the analysis of the identified quasi-stream vortices depending of tip-speed ratio, especially for small values, where the flow around the blades experiences massive stall.

Acknowledgments: The financial support of the Dirección de Investigaciones y Desarrollo Tecnológico of Universidad Autónoma de Occidente is gratefully acknowledged. This work was partially sponsored by the Young Researchers program from the Colombian administrative department of science, technology and innovation (Colciencias) and also by Vicerrectoria de Investigaciones of Universidad de los Andes. Collaboration of Pablo Cortés is also gratefully acknowledged.

Author Contributions: Santiago Laín wrote the paper, Simulations were conceived by Santiago Laín and Omar D. López and performed by Manuel A. Taborda. Analysis and discussion of the results were carried out by Santiago Laín, Manuel A. Taborda and Omar D. López. Santiago Laín wrote the paper.

Conflicts of Interest: The authors declare no conflict of interest.

\section{Appendix A}

Here, the behavior along a revolution of the skin friction coefficient and the skin friction lines is discussed for the case of symmetric winglet.

Figure A1 shows the skin friction lines pattern on the blades extrados (top row) and intrados (bottom row) along a turn of the turbine for the symmetric winglet configuration. Moreover, the surfaces are colored by the value of the skin friction coefficient which is the non-dimensional value associated to the wall shear stress.

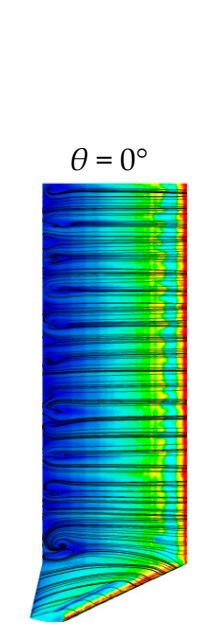

(a)

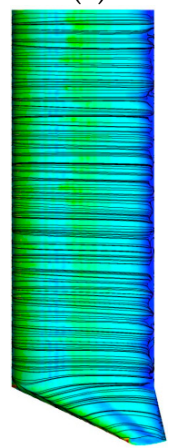

(g)

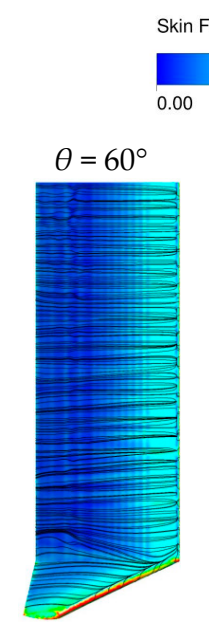

(b)

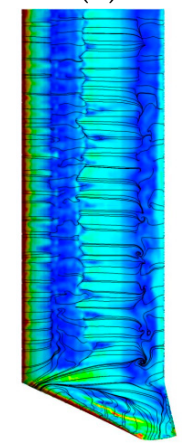

(h)

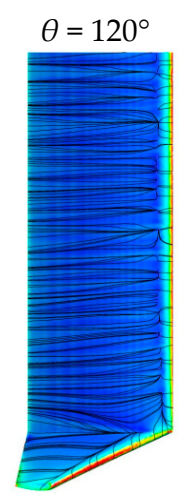

(c)

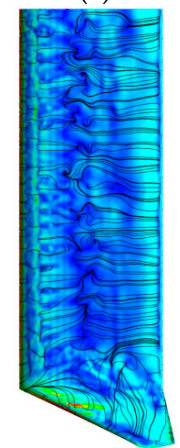

(i)

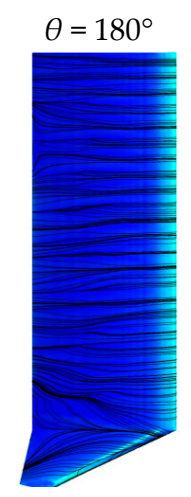

(d)

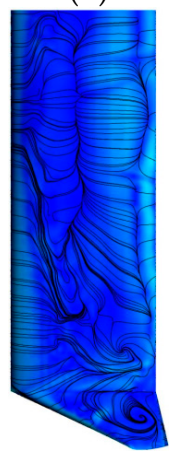

(j)

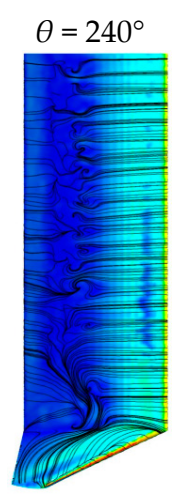

(e)

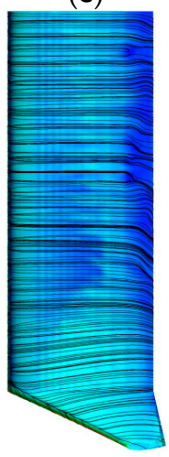

(k)

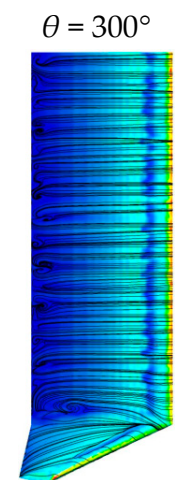

(f)

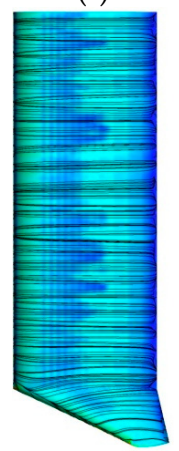

(1)

Figure A1. Skin friction coeficient and friction lines on the extrados (top row) and intrados (bottom row) for different angular positions along a turbine revolution. Blade with symmetric winglet. 
At $0^{\circ}$ angular position, the flow is attached in both surfaces, extrados and intrados, but near the trailing edge on the extrados some foci can be seen implying the generation of vortex cores that extend into the fluid. In both sides the transition from laminar to turbulent boundary layer can be appreciated because the skin friction coefficient, starting from the leading edge (LE) towards the trailing edge (TE), first decreases reaching light blue color and then increases again (green or even yellow color). The same conclusion can be drawn from Figure 15a looking at the intermittency contours. For positive azimuthal angles and in the upstream semicircle, Figure A1 indicates that boundary layer around extrados surface keeps laminar because the skin friction coefficient keeps a monotonically decreasing trend from LE to TE. However, the flow in the intrados experiences a more complex dynamics. At $\theta=60^{\circ}$, the laminar boundary layer develops, the skin friction decreases very fast and the flow detaches as indicated by the vertical separation line in Figure A1h, where the color changes from light to dark blue; then, flow reattaches in a vertical attachment line (color changes from dark to light blue or green); from that point a turbulent boundary layer develops and the flow is detached again in another vertical separation line close to the trailing edge. The flow in the intrados at $\theta=120^{\circ}$ shows the same three separation lines of Figure A1h but closer to the leading edge; at around $40 \%$ of the chord the boundary layer is completely detached with a bound vortex along the blade span and a trailing vortex in the winglet. At $\theta=180^{\circ}$ (Figure A1j) the boundary layer is fully detached along the intrados surface (see also Figure 12 right column) which is reflected in the smallest values of the skin friction coefficient; at this point the blade does not produce net torque.

In the downstream semicircle, the flow along the intrados surface tends to be progressive attached as the azimuthal angle increases up to $\theta=240^{\circ}$ where the flow is completely attached again (Figure A1k). From that point and up to the end of the cycle, the flow around the intrados keeps attached. Here, the transition from laminar to turbulent boundary layer can be observed due to the color change of the skin friction coefficient. On the other hand, the skin friction lines in the extrados present patterns with singular points and separation lines (e.g., see Figure A1e) up to $\theta=270^{\circ}$ although the boundary layer is not massively separated. From that angle, the flow maintains attached to the extrados, always presenting the boundary layer transition from laminar to turbulent regime.

\section{References}

1. López, O.; Meneses, D.; Quintero, B.; Laín, S. Computational study of transient flow around Darrieus type Cross Flow Water Turbines. J. Renew. Sustain. Energy 2016, 8, 014501. [CrossRef]

2. Trivedi, C.; Cervantes, M.J.; Dahlhaug, O.G. Experimental and numerical studies of a high-head Francis turbine: A review of the Francis-99 test case. Energies 2016, 9, 74. [CrossRef]

3. Trivedi, C.; Cervantes, M.J.; Gandhi, B.K. Investigation of a high head Francis turbine at runaway operating conditions. Energies 2016, 9, 149. [CrossRef]

4. Caballero, A.D.; Laín, S. A review on Computational Fluid Dynamics modelling in human thoracic aorta. Cardiovasc. Eng. Technol. 2013, 4, 103-130. [CrossRef]

5. Göz, M.F.; Laín, S.; Sommerfeld, M. Study of the numerical instabilities in Lagrangian tracking of bubbles and particles in two-phase flow. Comput. Chem. Eng. 2004, 28, 2727-2733. [CrossRef]

6. Sommerfeld, M.; Laín, S. From elementary processes to the numerical prediction of industrial particle-laden flows. Multiph. Flow Technol. 2009, 21, 123-140. [CrossRef]

7. Jin, X.; Zhao, G.; Gao, K.; Ju, W. Darrieus vertical axis wind turbine: Basic research methods. Renew. Sustain. Energy Rev. 2015, 42, 212-225. [CrossRef]

8. Howell, R.; Qin, N.; Edwards, J.; Durrani, N. Wind tunnel and numerical study of a small vertical axis wind turbine. Renew. Energy 2010, 35, 412-422. [CrossRef]

9. Untaroiu, A.; Wood, H.G.; Allaire, P.E.; Ribando, R.J. Investigation of Self-Sarting Capability of Vertical Axis Wind Turbines Using a Computational Fluid Dynamics Approach. J. Sol. Energy Eng. 2011, 133, 041010. [CrossRef]

10. Hill, N.; Dominy, R.; Ingram, G.; Dominy, J. Darrieus turbines: The physics of self-starting. Proc. Inst. Mech. Eng. Part A 2008, 223, 21-29. [CrossRef]

11. Castelli, M.R.; Benini, E. Effect of Blade Inclination Angle of a Darrieus Wind Turbine. J. Turbomach. 2012, 134, 031016. [CrossRef] 
12. Siddiqui, M.S.; Durrani, N.; Akhtar, I. Quantification of the effects of geometric approximations on the performance of a vertical axis wind turbine. Renew. Energy 2015, 74, 661-670. [CrossRef]

13. Laín, S.; Osorio, C. Simulation and evaluation of a straight-bladed Darrieus-type cross flow marine turbine. J. Sci. Ind. Res. 2010, 69, 906-912.

14. Dai, Y.M.; Lam, W. Numerical study of straight-bladed Darrieus-type tidal turbine. ICE-Energy 2009, 162, 67-76. [CrossRef]

15. Amet, E.; Maître, T.; Pellone, C.; Achard, J.L. 2D numerical simulations of blade-vortex interaction in a Darrieus turbine. J. Fluids Eng. 2009, 131, 111103. [CrossRef]

16. Maître, T.; Amet, E.; Pellone, C. Modeling of the flow in a Darrieus water turbine: Wall grid refinement analysis and comparison with experiments. Renew. Energy 2013, 51, 497-512. [CrossRef]

17. Amet, E. Simulation Numérique D'une Hydrolienne à Axe Vertical de Type Darrieus. Ph.D. Thesis, Institut Polytechnique de Grenoble, Grenoble, France, 2009.

18. Hall, T.J. Numerical Simulation of a Cross Flow Marine Hydrokinetic Turbine. Master's Thesis, University of Washington, Washington, DC, USA, 2012.

19. Pellone, C.; Maître, T.; Amet, E. 3D RANS modeling of a cross flow water turbine. In Advances in Hydroinformatics; Gourbesville, P., Cunge, J., Caignaert, G., Eds.; Springer: Singapore, 2014; pp. $405-418$. ISBN 978-9-81-287615-7.

20. Marsh, P.; Ranmuthugala, D.; Penesis, I.; Thomas, G. Numerical investigation of blade helicity on the performance characteristics of vertical axis tidal turbines. Renew. Energy 2015, 81, 926-935. [CrossRef]

21. Ferreira, C.S. The Near Wake of the VAWT, 2D and 3D Views of the VAWT Aerodynamics. Ph.D. Thesis, TU Delft, Delft, The Netherlands, 2009.

22. Yao, J.; Wang, J.; Yuan, W.; Wang, H.; Cao, L. Analysis on the influence of turbulence model changes to aerodynamic performance of vertical axis wind turbine. Procedia Eng. 2012, 31, 274-281. [CrossRef]

23. McNaughton, J.; Billard, F.; Revell, A. Turbulence modelling of low Reynolds number flow effects around a vertical axis turbine at a range of tip-speed ratios. J. Fluids Struct. 2017, 47, 124-138. [CrossRef]

24. Ghasemian, M.; Najafian Ashrafi, Z.; Sedaghat, A. A review on computational fluid dynamic simulation techniques for Darrieus vertical axis wind turbines. Energy Convers. Manag. 2017, 149, 87-100. [CrossRef]

25. Marsh, P.; Ranmuthugala, D.; Penesis, I.; Thomas, G. The influence of turbulence model and two and three-dimensional domain selection on the simulated performance characteristics of vertical axis tidal turbines. Renew. Energy 2017, 105, 106-116. [CrossRef]

26. Shamsoddin, S.; Porté-Agel, F. Large Eddy Simulation of Vertical Axis Wind Turbines wakes. Energies 2014, 7, 890-912. [CrossRef]

27. Shamsoddin, S.; Porté-Agel, F. A Large Eddy Simulation study of Vertical Axis Wind Turbines wakes in the Atmospheric Boundary Layer. Energies 2016, 9, 366. [CrossRef]

28. Hezaveh, S.H.; Bou-Zeid, E.; Lohry, M.W.; Martinelli, L. Simulation and wake analysis of a single vertical axis wind turbine. Wind Energy 2017, 20, 713-730. [CrossRef]

29. Johansen, J.; Sørensen, N.N. Numerical analysis of winglets on wind turbine blades using CFD. In Proceedings of the European Wind Energy Conference and Exhibition, Milan, Italy, 7-10 May 2007; pp. 1184-1189.

30. Ferrer, E.; Munduate, X. Wind turbine blade tip comparison using CFD. J. Phys. Conf. Ser. 2007, 75, 012005. [CrossRef]

31. Liang, Y.; Zhang, L.; Li, E.; Liu, X.; Yang, Y. Design Considerations of Rotor Configuration for Straight-Bladed Vertical Axis Wind Turbines. Adv. Mech. Eng. 2014, 6, 534906. [CrossRef]

32. Zhu, B.; Sun, X.; Wang, Y.; Huang, D. Performance characteristics of a horizontal axis wind turbine with fusion winglet. Energy 2017, 120, 431-440. [CrossRef]

33. Islam, M.; Fartaj, A.; Carriveau, R. Analysis of the Design Parameters related to a Fixed-pitch Straight Bladed Vertical Axis Wind Turbine. Wind Eng. 2008, 32, 491-507. [CrossRef]

34. Sekiya, K.; Ueki, Y.; Nishizawa, Y.; Ushiyama, I.; Suzuki, M.; Taniguchi, H. A study on the improvement of the performance of straight bladed vertical axis wind turbine. In Proceedings of the Renewable Energy Conference 2010, Yokohama, Japan, 27 June-2 July 2010.

35. Ahmed, N.A.; Netto, K.J. Computer Aided Design and Manufacture of a Novel Vertical Axis Wind Turbine Rotor with Winglet. Appl. Mech. Mater. 2014, 607, 581-587. [CrossRef]

36. Amato, F.; Bedon, G.; Castelli, M.; Benini, E. Numerical Analysis of the Influence of Tip Devices on the Power Coefficient of a VAWT. Int. J. Aerosp. Mech. Eng. 2013, 7, 390-397. 
37. Li, Y.; Calisal, S.M. Three-dimensional effects and arm effects on modeling a vertical axis tidal current turbine. Renew. Energy 2010, 35, 2325-2334. [CrossRef]

38. Heyson, H.H.; Riebe, G.D.; Fulton, C.L. Theoretical Parametric Study of the Relative Advantages of Winglets and Wing-Tip Extensions; NASA Technical Paper 1020; NASA Langley Research Center: Hampton, VA, USA, 1977.

39. Gupta, A.; Amano, R.S. CFD of wind turbine blade with winglets. In Proceedings of the ASME 2012 International Design Engineering Technical Conferences \& Computers and Information in Engineering Conference, IDETC/CIE 2012, Chicago, IL, USA, 12-15 August 2012.

40. Mc Cormick, B.W. Aerodynamics, Aeronautics and Flight Mechanics, 2nd ed.; John Wiley \& Sons: New York, NY, USA, 1994; ISBN 978-0-47-157506-1.

41. Masak, P.C. Design of Winglets for Sailplanes. Available online: http:/ / soaringweb.org/Soaring_Index/ 1993/1993_issue.html (accessed on 10 September 2017).

42. Rajendran, S. Design of Parametric Winglets and Wing Tip Devices: A Conceptual Design Approach. Master's Thesis, Linkoping University, Linkoping, Sweden, 2012.

43. Langtry, R.B.; Menter, F.R. Correlation-based transition modeling for unstructured parallelized computational fluid dynamics codes. AIAA J. 2009, 47, 2894-2906. [CrossRef]

44. Langtry, R.B. A Correlation Based Transition Model Using Local Variables for Unstructured Parallelized CFD Codes. Ph.D. Thesis, University Stuttgart, Stuttgart, Germany, 2006.

45. Dai, Y.M.; Gardiner, N.; Sutton, R.; Dyson, P.K. Hydrodynamic analysis models for the design of Darrieus-type vertical-axis marine current turbines. Proc. Inst. Mech. Eng. Part M J. Eng. Marit. Environ. 2011, 225, 295-307. [CrossRef]

46. Balduzzi, F.; Bianchini, A.; Malece, R.; Ferrara, G.; Ferrari, L. Critical issues in the CFD simulation of Darrieus wind Turbines. Renew. Energy 2016, 85, 419-435. [CrossRef]

47. Yakhot, Y.; Orszag, S.A.; Thangam, S.; Gatski, T.B.; Speziale, C.G. Development of turbulence models for shear flows by a double expansion technique. Phys. Fluids A 1992, 4, 1510-1520. [CrossRef]

48. Menter, F.J. Two-Equation Eddy-Viscosity Turbulence Models for Engineering Applications. AIAA. J. 1994, 32, 269-289. [CrossRef]

49. Launder, B.E.; Reece, G.J.; Rodi, W. Progress in the Development of a Reynolds-stress Turbulence Closure. J. Fluid Mech. 1975, 68, 537-566. [CrossRef]

50. Tobak, M.; Peak, D.J. Topology of three-dimensional separated flows. Annu. Rev. Fluid Mech. 1982, 14, 61-85. [CrossRef]

51. Dick, E.; Kubacki, S. Transition models for turbomachinery boundary layer flows: A review. Int. J. Turbomach. Propuls. Power 2017, 2, 4. [CrossRef] 